\title{
Which Is the Worst-Case Nash Equilibrium?^
}

\author{
Thomas Lücking $^{1}$, Marios Mavronicolas ${ }^{2}$, Burkhard Monien ${ }^{1}$, \\ Manuel Rode ${ }^{1, \star \star}$, Paul Spirakis ${ }^{3,4}$, and Imrich Vrto ${ }^{5}$ \\ 1 Faculty of Computer Science, Electrical Engineering and Mathematics \\ University of Paderborn, Fürstenallee 11, 33102 Paderborn, Germany \\ \{luck, bm, rode\}@uni-paderborn. de \\ 2 Department of Computer Science, University of Cyprus \\ P. O. Box 20537, Nicosia CY-1678, Cyprus \\ mavronic@ucy.ac.cy \\ 3 Computer Technology Institute, P. O. Box 1122, 26110 Patras, Greece \\ spirakis@cti.gr \\ 4 Department of Computer Engineering and Informatics \\ University of Patras, Rion, 26500 Patras, Greece \\ 5 Institute of Mathematics, Slovak Academy of Sciences \\ 84104 Bratislava 4, Dúbravskaá 9, Slovak Republic \\ imrich@ifi.savba.sk
}

\begin{abstract}
A Nash equilibrium of a routing network represents a stable state of the network where no user finds it beneficial to unilaterally deviate from its routing strategy. In this work, we investigate the structure of such equilibria within the context of a certain game that models selfish routing for a set of $n$ users each shipping its traffic over a network consisting of $m$ parallel links. In particular, we are interested in identifying the worst-case Nash equilibrium - the one that maximizes social cost. Worst-case Nash equilibria were first introduced and studied in the pioneering work of Koutsoupias and Papadimitriou [9].

More specifically, we continue the study of the Conjecture of the Fully Mixed Nash Equilibrium, henceforth abbreviated as FMNE Conjecture, which asserts that the fully mixed Nash equilibrium, when existing, is the worst-case Nash equilibrium. (In the fully mixed Nash equilibrium, the mixed strategy of each user assigns (strictly) positive probability to every link.) We report substantial progress towards identifying the validity, methodologies to establish, and limitations of, the FMNE Conjecture.
\end{abstract}

\section{Introduction}

Motivation and Framework. Nash equilibrium [12,13] is arguably the most important solution concept in (non-cooperative) Game Theory ${ }^{1}$. It represents

* This work has been partially supported by the IST Program of the European Union under contract numbers IST-1999-14186 (ALCOM-FT) and IST-2001-33116 (FLAGS), by funds from the Joint Program of Scientific and Technological Collaboration between Greece and Cyprus, by research funds at University of Cyprus, and by the VEGA grant No. 2/3164/23.

** Graduate School of Dynamic Intelligent Systems

${ }^{1}$ See [14] for a concise introduction to contemporary Game Theory. 
a stable state of the play of a strategic game in which each player holds an accurate opinion about the (expected) behavior of other players and acts rationally. Understanding the combinatorial structure of Nash equilibria is a necessary prerequisite to either designing efficient algorithms to compute them, or establishing corresponding hardness and thereby designing (efficient) approximation algorithms ${ }^{2}$.

In this work, we embark on a systematic study of the combinatorial structure of Nash equilibria in the context of a simple routing game that models selfish routing over a non-cooperative network such as the Internet. This game was originally introduced in a pioneering work of Koutsoupias and Papadimitriou [9]; that work defined coordination ratio (also known as price of anarchy [15]) as a worst-case measure of the impact of the selfish behavior of users on the efficiency of routing over a non-cooperative network operating at a Nash equilibrium. As a worst-case measure, the coordination ratio bounds the maximum loss of efficiency due to selfish behavior of users at the worst-case Nash equilibrium; in sharp contrast, the principal motivation of our work is to identify the actual worst-case Nash equilibrium of the selfish routing game.

Within the framework of the selfish routing game of Koutsoupias and Papadimitriou [9], we assume a collection of $n$ users, each employing a mixed strategy, which is a probability distribution over $m$ parallel links, to control the shipping of its own assigned traffic. For each link, a capacity specifies the rate at which the link processes traffic. In a Nash equilibrium, each user selfishly routes its traffic on those links that minimize its expected latency cost, given the network congestion caused by the other users. The social cost of a Nash equilibrium is the expectation, over all random choices of the users, of the maximum, over all links, latency through a link. The worst-case Nash equilibrium is one that maximizes social cost.

Our study distinguishes between pure Nash equilibria, where each user chooses exactly one link (with probability one), and mixed Nash equilibria, where the choices of each user are modeled by a probability distribution over links. Of special interest to our work is the fully mixed Nash equilibrium [10], where each user chooses each link with non-zero probability; henceforth, denote $\mathbf{F}$ the fully mixed Nash equilibrium. We will also introduce and study disjointly mixed Nash equilibria, where (loosely speaking) mixed strategies of different users do not intersect.

Allowing link capacities to vary arbitrarily gives rise to the standard model of related links, also known as model of uniform links in the scheduling literature (cf. Gonzales et al. [5]); the name is due to the fact that the order of the delays a user experiences on each of the links is the same across all users. A special case of the model of related links is the model of identical links, where all link capacities are equal (cf. Graham [6]); thus, in this model, each user incurs the same delay on all links. We also consider the model of unrelated links, where instead of associating a traffic and a capacity with each user and link, respec-

\footnotetext{
${ }^{2}$ Computation of Nash equilibria has been long observed to be a very challenging, yet notoriously hard algorithmic problem; see [15] for an advocation.
} 
tively, we assign a delay for each pair of a user and a link in an arbitrary way (cf. Horowitz and Sahni [7]); thus, in the unrelated links model, there is no relation between the delays incurred to a user on different links. Reciprocally, in the model of identical traffics, all user traffics are equal; they may vary arbitrarily in the model of arbitrary traffics. We are interested in understanding the impact of model assumptions on links and users on the patterns of the worst-case Nash equilibria for the selfish routing game we consider.

Results and Contribution. In this work, we embark on a systematic study of a natural conjecture due to Gairing et al. [4], which asserts that the fully mixed Nash equilibrium is the worst-case Nash equilibrium (with respect to social cost).

\section{Fully Mixed Nash Equilibrium Conjecture [4].}

Consider the model of arbitrary traffics and related links. Then, for any traffic vector $\mathbf{w}$ such that the fully mixed Nash equilibrium $\mathbf{F}$ exists, and for any Nash equilibrium $\mathbf{P}, \mathrm{SC}(\mathbf{w}, \mathbf{P}) \leq \mathrm{SC}(\mathbf{w}, \mathbf{F})$.

Henceforth, abbreviate the Fully Mixed Nash Equilibrium Conjecture as the FMNE Conjecture. Our study reports substantial progress towards the settlement of the FMNE Conjecture:

- We prove the FMNE Conjecture for several interesting special cases of it (within the model of related links).

- In doing so, we provide proof techniques and tools which, while applicable to interesting special cases of it, may suffice for the general case as well.

- We reveal limitations of the FMNE Conjecture by establishing that it is not, in general, valid over the model of unrelated links; we present both positive and negative instances for the conjecture.

Related Work, Comparison and Significance. The selfish routing game considered in this paper was first introduced and studied in the pioneering work of Koutsoupias and Papadimitriou [9]. This game was subsequently studied in the work of Mavronicolas and Spirakis [10], where fully mixed Nash equilibria were introduced and analyzed. Both works focused mainly on proving bounds on coordination ratio. Subsequent works that provided bounds on coordination ratio include $[1,2,8]$. The work of Fotakis et al. [3] was the first to study the combinatorial structure and the computational complexity of Nash equilibria for the selfish routing game we consider; that work was subsequently extended by Gairing et al. [4]. (See details below.)

The closest to our work are the one by Fotakis et al. [3] and the one by Gairing et al. [4].

- The FMNE Conjecture has been inspired by two results due to Fotakis et al. [3] that confirm or support the conjecture. First, Fotakis et al. [3, Theorem 6] establish the Fully Mixed Nash Equilibrium Conjecture for the model of identical links and assuming that $n=2$; Theorem 3 in this work extends this 
result to the model of related links, still assuming that $n=2$ while assuming, in addition, that traffics are identical. Second, Fotakis et al. [3, Theorem 7] prove that, for the model of related links and of identical traffics, the social cost of any Nash equilibrium is no more than 49.02 times the social cost of the fully mixed Nash Equilibrium.

- The FMNE Conjecture was explicitly stated in the work of Gairing et al. [4, Conjecture 1.1]. In the same paper, two results are shown that confirm or support the conjecture. First, Gairing et al. [4, Theorem 4.2] establish the validity of the FMNE Conjecture when restricted to pure Nash equilibria. Second, Gairing et al. [4, Theorem 5.1] prove that for the model of identical links, the social cost of any Nash equilibrium is no more than $6+\varepsilon$ times the social cost of the fully mixed Nash equilibrium, for any constant $\varepsilon>0$. (Note that since this result does not assume identical traffics, it is incomparable to the related result by Fotakis et al. [3, Theorem 7] (for the model of related links) which does.)

The ultimate settlement of the FMNE Conjecture (for the model of related links) may reveal an interesting complexity-theoretic contrast between the worstcase pure and the worst-case mixed Nash equilibria. On one hand, identifying the worst-case pure Nash equilibrium is an $\mathcal{N} \mathcal{P}$-hard problem [3, Theorem 4]; on the other hand, if the FMNE Conjecture is valid, identification of the worstcase mixed Nash equilibrium is immediate in the cases where the fully mixed Nash equilibrium exists. (In addition, the characterization of the fully mixed Nash equilibrium shown in [10, Theorem 14] implies that such existence can be checked in polynomial time.)

Road Map. The rest of this paper is organized as follows. Section 2 presents our definitions and some preliminaries. The case of disjointly mixed Nash equilibria is treated in Section 3. Section 4 considers the case of identical traffics and related links with $n=2$. The reciprocal case of identical traffics and identical links with $m=2$ is studied in Section 5. Section 6 examines the case of unrelated links. We conclude, in Section 7, with a discussion of our results and some open problems.

\section{Framework}

Most of our definitions are patterned after those in [10, Section 2], [3, Section 2] and [4, Section 2], which, in turn, were based on those in [9, Sections 1 \& 2].

Mathematical Preliminaries and Notation. Throughout, denote for any integer $m \geq 2,[m]=\{1, \ldots, m\}$. For a random variable $X$, denote $\mathcal{E}(X)$ the expectation of $X$.

General. We consider a network consisting of a set of $m$ parallel links $1,2, \ldots, m$ from a source node to a destination node. Each of $n$ network users $1,2, \ldots, n$, or users for short, wishes to route a particular amount of traffic along a (non-fixed) link from source to destination. (Throughout, we will be using subscripts for users and superscripts for links.) In the model of related links, denote $w_{i}$ the 
traffic of user $i \in[n]$, and $W=\sum_{i \in[n]} w_{i}$. Define the $n \times 1$ traffic vector $\mathbf{w}$ in the natural way. Assume throughout that $m>1$ and $n>1$. Assume also, without loss of generality, that $w_{1} \geq w_{2} \geq \ldots \geq w_{n}$. In the model of unrelated links, denote $C_{i j}$ the cost of user $i \in[n]$ on link $j \in[m]$. Define the $n \times m$ cost matrix $\mathbf{C}$ in the natural way.

A pure strategy for user $i \in[n]$ is some specific link. A mixed strategy for user $i \in[n]$ is a probability distribution over pure strategies; thus, a mixed strategy is a probability distribution over the set of links. The support of the mixed strategy for user $i \in[n]$, denoted support $(i)$, is the set of those pure strategies (links) to which $i$ assigns positive probability. A pure strategy profile is represented by an $n$-tuple $\left\langle\ell_{1}, \ell_{2}, \ldots, \ell_{n}\right\rangle \in[m]^{n}$; a mixed strategy profile is represented by an $n \times m$ probability matrix $\mathbf{P}$ of $n m$ probabilities $p_{i}^{j}, i \in[n]$ and $j \in[m]$, where $p_{i}^{j}$ is the probability that user $i$ chooses link $j$.

For a probability matrix $\mathbf{P}$, define indicator variables $I_{i}^{j} \in\{0,1\}$, where $i \in[n]$ and $j \in[m]$, such that $I_{i}^{j}=1$ if and only if $p_{i}^{j}>0$. Thus, the support of the mixed strategy for user $i \in[n]$ is the set $\left\{j \in[m] \mid I_{i}^{j}=1\right\}$. For each link $j \in[m]$, define the view of link $j$, denoted view $(j)$, as the set of users $i \in[n]$ that potentially assign their traffics to link $j$; so, view $(j)=\left\{i \in[n] \mid I_{i}^{j}=1\right\}$. For each link $j \in[m]$, denote $V^{j}=\mid$ view $(j) \mid$.

Syntactic Classes of Mixed Strategies. A mixed strategy profile $\mathbf{P}$ is disjointly mixed if for all links $j \in[m],\left|\left\{i \in \operatorname{view}(j): p_{i}^{j}<1\right\}\right| \leq 1$, that is, there is at most one non-pure user on each link. A mixed strategy profile $\mathbf{P}$ is fully mixed [10, Section 2.2] if for all users $i \in[n]$ and links $j \in[m], I_{i}^{j}=1^{3}$. Throughout, we will cast a pure strategy profile as a special case of a mixed strategy profile in which all (mixed) strategies are pure.

System, Models and Cost Measures. In the model of related links, denote $c^{\ell}>0$ the capacity of link $\ell \in[m]$, representing the rate at which the link processes traffic, and $C=\sum_{l \in[m]} c^{l}$. So, the latency for traffic $w$ through link $\ell$ equals $w / c^{\ell}$. In the model of identical capacities, all link capacities are equal to $c$, for some constant $c>0$; link capacities may vary arbitrarily in the model of arbitrary capacities. Assume throughout, without loss of generality, that $c^{1} \geq$ $c^{2} \geq \ldots \geq c^{m}$. In the model of identical traffics, all user traffics are equal to 1 ; user traffics may vary arbitrarily in the model of arbitrary traffics.

For a pure strategy profile $\left\langle\ell_{1}, \ell_{2}, \ldots, \ell_{n}\right\rangle$, the latency cost for user $i \in[n]$, denoted $\lambda_{i}$, is the latency cost of the link it chooses, that is, $\left(\sum_{k: \ell_{k}=\ell_{i}} w_{k}\right) / c^{\ell_{i}}$. For a mixed strategy profile $\mathbf{P}$, denote $\delta^{\ell}$ the actual traffic on link $\ell \in[\mathrm{m}]$; so, $\delta^{\ell}$ is a random variable. For each link $\ell \in[m]$, denote $\theta^{\ell}$ the expected traffic on link $\ell \in[m]$; thus, $\theta^{\ell}=\mathcal{E}\left(\delta^{\ell}\right)=\sum_{i=1}^{n} p_{i}^{\ell} w_{i}$. For a mixed strategy profile $\mathbf{P}$, the expected latency cost for user $i \in[n]$ on link $\ell \in[m]$, denoted $\lambda_{i}^{\ell}$, is the expectation, over all random choices of the remaining users, of the latency cost for user $i$ had its traffic been assigned to link $\ell$; thus,

\footnotetext{
${ }^{3}$ An earlier treatment of fully mixed strategies in the context of bimatrix games has been found in [16], called there completely mixed strategies. See also [11] for a subsequent treatment in the context of strategically zero-sum games.
} 


$$
\lambda_{i}^{\ell}=\frac{w_{i}+\sum_{k=1, k \neq i} p_{k}^{\ell} w_{k}}{c^{\ell}}=\frac{\left(1-p_{i}^{\ell}\right) w_{i}+\theta^{\ell}}{c^{\ell}} .
$$

For each user $i \in[n]$, the minimum expected latency cost, denoted $\lambda_{i}$, is the minimum, over all links $\ell \in[m]$, of the expected latency cost for user $i$ on link $\ell$; thus, $\lambda_{i}=\min _{\ell \in[m]} \lambda_{i}^{\ell}$.

Associated with a traffic vector $\mathbf{w}$ and a mixed strategy profile $\mathbf{P}$ is the social cost [9, Section 2], denoted $\mathrm{SC}(\mathbf{w}, \mathbf{P})$, which is the expectation, over all random choices of the users, of the maximum (over all links) latency of traffic through a link; thus,

$\mathrm{SC}(\mathbf{w}, \mathbf{P})=\mathcal{E}\left(\max _{\ell \in[m]} \frac{\sum_{k: \ell_{k}=\ell} w_{k}}{c^{\ell}}\right)=\sum_{\left\langle\ell_{1}, \ell_{2}, \ldots, \ell_{n}\right\rangle \in[m]^{n}}\left(\prod_{k=1}^{n} p_{k}^{\ell_{k}} \cdot \max _{\ell \in[m]} \frac{\sum_{k: \ell_{k}=\ell} w_{k}}{c^{\ell}}\right)$.

Note that SC $(\mathbf{w}, \mathbf{P})$ reduces to the maximum latency through a link in the case of pure strategies. On the other hand, the social optimum [9, Section 2] associated with a traffic vector $\mathbf{w}$, denoted OPT $(\mathbf{w})$, is the least possible maximum (over all links) latency of traffic through a link. Note that while $\mathbf{S C}(\mathbf{w}, \mathbf{P})$ is defined in relation to a mixed strategy profile $\mathbf{P}, \mathrm{OPT}(\mathbf{w})$ refers to the optimum pure strategy profile.

In the model of unrelated links, the latency of user $i$ on link $l$ is its cost $C_{i l}$. Thus, the expected latency cost of user $i$ on link $l$ translates to $\lambda_{i}^{l}=C_{i l}+$ $\sum_{k=1, k \neq i} p_{i}^{l} C_{k l}$, and the social cost, now depending on $\mathbf{C}$ and the strategy profile $\mathbf{P}$, is defined by $\mathrm{SC}(\mathbf{C}, \mathbf{P})=\sum_{\left\langle l_{1}, l_{2}, \ldots, l_{n}\right\rangle \in[m]^{n}}\left(\prod_{k=1}^{n} p_{k}^{l_{k}} \cdot \max _{l \in[m]} \sum_{k: l_{k}=l} C_{k l}\right)$.

Nash Equilibria. We are interested in a special class of mixed strategies called Nash equilibria [13] that we describe below. Formally, the probability matrix $\mathbf{P}$ is a Nash equilibrium [9, Section 2] if for all users $i \in[n]$ and links $\ell \in[m]$, $\lambda_{i}^{\ell}=\lambda_{i}$ if $I_{i}^{\ell}=1$, and $\lambda_{i}^{\ell} \geq \lambda_{i}$ if $I_{i}^{\ell}=0$. Thus, each user assigns its traffic with positive probability only on links for which its expected latency cost is minimized; this implies that there is no incentive for a user to unilaterally deviate from its mixed strategy in order to avoid links on which its expected latency cost is higher than necessary. The coordination ratio [9] is the maximum value, over all traffic vectors $\mathbf{w}$ and Nash equilibria $\mathbf{P}$ of the ratio $\mathrm{SC}(\mathbf{w}, \mathbf{P}) / \mathrm{OPT}(\mathbf{w})$. In the model of unrelated links, the coordination ratio translates to the maximum value of $\mathrm{SC}(\mathbf{C}, \mathbf{P}) / \mathrm{OPT}(\mathbf{C})$.

Mavronicolas and Spirakis [10, Lemma 15] show that in the model of identical links, all links are equiprobable in a fully mixed Nash equilibrium.

Lemma 1 (Mavronicolas and Spirakis [10]). Consider the fully mixed case under the model of identical capacities. Then, there exists a unique Nash equilibrium with associated Nash probabilities $p_{i}^{\ell}=1 / m$, for any user $i \in[n]$ and link $\ell \in[m]$.

Gairing et al. [4, Lemma 4.1] show that in the model of related links, the minimum expected latency cost of any user $i \in[n]$ in a Nash equilibrium $\mathbf{P}$ is bounded by its minimum expected latency cost in the fully mixed Nash equilibrium $\mathbf{F}$. 
Lemma 2 (Gairing et al. [4]). Fix any traffic vector $\mathbf{w}$, mixed Nash equilibrium $\mathbf{P}$ and user $i$. Then, $\lambda_{i}(\mathbf{w}, \mathbf{P}) \leq \lambda_{i}(\mathbf{w}, \mathbf{F})$.

\section{Disjointly Mixed versus Fully Mixed Nash Equilibria}

In this section, we restrict ourselves to the case of disjointly mixed Nash equilibria, and we establish the FMNE Conjecture for this case. We prove:

Theorem 1. Fix any traffic vector $\mathbf{w}$ such that $\mathbf{F}$ exists, and any disjointly mixed Nash equilibrium $\mathbf{P}$. Then, $\mathrm{SC}(\mathbf{w}, \mathbf{P}) \leq \mathrm{SC}(\mathbf{w}, \mathbf{F})$.

Corollary 1. Consider the model of related links, and assume that $n=2$ and $m=2$. Then, the FMNE Conjecture is valid.

\section{Identical Traffics, Related Links and $n=2$}

In this section we restrict to 2 users with identical traffics, that is, $w_{1}=w_{2}$. Without loss of generality we assume $w_{1}=w_{2}=1$ and $c^{1} \geq \cdots \geq c^{m}$. In the following, we denote by support(1) and support(2) the supports of user 1 and 2 , respectively, and by $p_{i}^{j}$ and $f_{i}^{j}$ the probabilities for user $i$ to choose link $j$ in $\mathbf{P}$ and $\mathbf{F}$, respectively. Since we consider two users with identical traffics, we have $f_{1}^{j}=f_{2}^{j}$ for all $j \in[m]$, and we write $f^{j}=f_{i}^{j}$.

In order to prove the FMNE Conjecture for this type of Nash equilibria we will use the following formula for the social cost of any Nash equilibrium $\mathbf{P}$ in this setting.

Theorem 2. In case of two users with identical traffics on $m$ related links, the social cost of any Nash equilibrium $\mathbf{P}$ is

$$
\mathrm{SC}(\mathbf{w}, \mathbf{P})=\lambda_{2}(\mathbf{P})+\sum_{1 \leq i<j \leq m} p_{2}^{i} p_{1}^{j}\left(\frac{1}{c^{j}}-\frac{1}{c^{i}}\right) .
$$

We now show that we only have to consider Nash equilibria $\mathbf{P}$ of certain structure.

Lemma 3. For any Nash equilibrium $\mathbf{P} \neq \mathbf{F}$ of two users with identical traffics on $m$ related links the following holds:

1. The supports of the two users are

$$
\operatorname{support}(1)=[r] \cup I_{1} \quad \text { and } \quad \operatorname{support}(2)=[r] \cup I_{2},
$$

where $I_{1}, I_{2}$ are disjoint sets of links not containing a link $i \in[r]$, such that

$$
[r] \cup I_{1} \cup I_{2}=\left[r+\left|I_{1}\right|+\left|I_{2}\right|\right] .
$$

2. All links in $I_{1}\left(I_{2}\right)$ have the same capacity. 
In order to prove the FMNE Conjecture for two users with identical traffics on $m$ related links in Theorem 3, we show that the following lemma holds.

Lemma 4. Let $\mathbf{G}$ be the fully mixed Nash equilibrium of two users with identical traffics on $m$ related links with capacities $c^{1} \geq \ldots \geq c^{m}$. Furthermore, let the last $s \geq 1$ links have the same capacity, and let $\mathbf{F}$ be the fully mixed Nash equilibrium of the instance received by increasing the capacities of the last $s$ links to $c^{m-s}$. Then $\mathrm{SC}(\mathbf{w}, \mathbf{F}) \leq \mathrm{SC}(\mathbf{w}, \mathbf{G})$.

Theorem 3. Consider the model of identical traffics and related links, and assume that $n=2$. Then, the FMNE Conjecture is valid.

\section{Identical Traffics, Identical Links and $m=2$}

We show:

Theorem 4. Consider the model of identical traffics and identical links, and assume that $m=2$ and $n$ is even. Then, the FMNE Conjecture is valid.

Proof. Since both the traffics and the link capacities are identical, we can assume without loss of generality that $w_{i}=1$ for all $i \in[n]$ and $c^{j}=1$ for all $j \in[m]$. Recall that in the case of identical capacities, the fully mixed Nash equilibrium $\mathbf{F}$ exists always (that is, for all traffic vectors $\mathbf{w})$. Hence, we will show that for any other Nash equilibrium $\mathbf{P}, \mathbf{S C}(\mathbf{w}, \mathbf{P}) \leq \mathbf{S C}(\mathbf{w}, \mathbf{F})$. Fix any Nash equilibrium $\mathbf{P}$. We can identify three sets of users in $\mathbf{P}: \mathcal{U}_{1}=\{i: \operatorname{support}(i)=\{1\}\}$, $\mathcal{U}_{2}=\{i: \operatorname{support}(i)=\{2\}\}$ and $\mathcal{U}_{12}=\{i: \operatorname{support}(i)=\{1,2\}\}$. There are $u=\min \left(\left|\mathcal{U}_{1}\right|,\left|\mathcal{U}_{2}\right|\right)$ (pure) users, which choose link 1 and link 2, respectively, with probability 1 . Therefore, $\mathrm{SC}(\mathbf{w}, \mathbf{P})=\mathrm{SC}\left(\mathbf{w}, \mathbf{P}^{\prime}\right)+u$, where $\mathbf{P}^{\prime}$ is the Nash equilibrium derived from $\mathbf{P}$ by omitting those $2 u$ users. We will show, that $\mathrm{SC}\left(\mathbf{w}, \mathbf{F}^{\prime}\right) \geq \mathrm{SC}\left(\mathbf{w}, \mathbf{P}^{\prime}\right)$ for the fully mixed Nash equilibrium $\mathbf{F}^{\prime}$ of $n-2 u$ users. As $\mathrm{SC}(\mathbf{w}, \mathbf{F})>\mathrm{SC}\left(\mathbf{w}, \mathbf{F}^{\prime}\right)+2 u$ (Lemma 5), this will prove the theorem.

Without loss of generality, we can assume that $\mathbf{P}^{\prime}$ is of the following form: $r$ (pure) users go on link 1 with probability 1 , and $n-r$ users choose both links with positive probability. We write $\mathbf{P}_{\mathbf{r}}$ for this kind of Nash equilibrium.

Lemma 5. For the fully mixed Nash equilibrium $\mathbf{F}$,

$$
\mathrm{SC}(\mathbf{w}, \mathbf{F})=\frac{n}{2}+\frac{n}{2^{n}}\left(\begin{array}{c}
n-1 \\
\frac{n}{2}-1
\end{array}\right)
$$

Lemma 6. For the Nash equilibrium $\mathbf{P}_{r}$ with two sets of users $\mathcal{U}_{1}=\{i$ : $\operatorname{support}(i)=\{1\}\}$ and $\mathcal{U}_{12}=\{i: \operatorname{support}(i)=\{1,2\}\}$ with $\left|\mathcal{U}_{1}\right|=r<n$ and $\left|\mathcal{U}_{12}\right|=n-r$ the Nash probabilities are

$$
p:=p_{i}^{1}=\frac{1}{2}-\frac{r}{2(n-r-1)}, \quad \text { and } \quad q:=p_{i}^{2}=\frac{1}{2}+\frac{r}{2(n-r-1)},
$$

for all users $i \in \mathcal{U}_{12}$. Furthermore, $n>2 r+1$ holds. 
Lemma 7. The social cost of the Nash equilibrium $\mathbf{P}_{r}$ is given by

$$
\begin{aligned}
\mathrm{SC}\left(\mathbf{w}, \mathbf{P}_{r}\right)= & \frac{n}{2}\left(\begin{array}{c}
n-r \\
\frac{n}{2}-r
\end{array}\right) p^{\frac{n}{2}-r} q^{\frac{n}{2}}+\sum_{i=\frac{n}{2}+1}^{n} i \cdot\left(\begin{array}{c}
n-r \\
i-r
\end{array}\right) p^{i-r} q^{n-i} \\
& +\sum_{i=\frac{n}{2}+1}^{n-r} i \cdot\left(\begin{array}{c}
n-r \\
i
\end{array}\right) p^{n-r-i} q^{i} .
\end{aligned}
$$

The proof is completed by showing that $\Delta:=\mathrm{SC}(\mathbf{w}, \mathbf{F})-\mathrm{SC}\left(\mathbf{w}, \mathbf{P}_{r}\right) \geq 0$.

\section{$6 \quad$ Unrelated Links}

In this section, we consider the case of unrelated links. We prove

Proposition 1. Consider the model of unrelated links. Fix any cost matrix $\mathbf{C}$ for which $\mathbf{F}$ exists, and a pure Nash equilibrium $\mathbf{P}$. Assume that $n \leq m$. Then, for any user $i, \lambda_{i}(\mathbf{P})<\lambda_{i}(\mathbf{F})$.

Theorem 5. Consider the model of unrelated links. Assume that $n \leq m$. Consider any cost matrix $\mathbf{C}$ such that the fully mixed Nash equilibrium $\mathbf{F}$ exists, and any pure Nash equilibrium $\mathbf{P}$. Then, $\mathrm{SC}(\mathbf{C}, \mathbf{P}) \leq \mathrm{SC}(\mathbf{C}, \mathbf{F})$.

Proof. Clearly, the social cost of any pure Nash equilibrium $\mathbf{P}$ is equal to the selfish cost of some user, while the social cost of a fully mixed Nash equilibrium $\mathbf{F}$ is at least the selfish cost of any user. Hence, Proposition 1 implies the claim.

Proposition 2. Consider the model of unrelated links. Assume that $n=2$. Fix any cost matrix $\mathbf{C}$ for which $\mathbf{F}$ exists, and any Nash equilibrium $\mathbf{P}$. Then, for any user $i \in[2], \lambda_{i}(\mathbf{P}) \leq \lambda_{i}(\mathbf{F})$.

Theorem 6. Consider the model of unrelated links. Assume that $n=2$ and $m=2$. Then, the FMNE Conjecture is valid.

We remark that Theorem 6 generalizes Corollary 1 to the case of unrelated links. We finally prove:

Theorem 7 (Counterexample to the FMNE Conjecture). Consider the model of unrelated links. Then, the FMNE Conjecture is not valid even if $n=3$ and $m=2$.

\section{Conclusion and Directions for Further Research}

We have verified the FMNE Conjecture over several interesting restrictions of the selfish routing game we considered for the case of related links. We have also investigated the FMNE Conjecture in the case of unrelated links, for which we have identified instances of the game that validate and falsify the FMNE Conjecture, respectively. The most obvious problem left open by our work is to 
establish the FMNE Conjecture in its full generality for the case of related links. We hope that several of the combinatorial techniques introduced in this work for settling special cases of the conjecture may be handy for the general case.

The FMNE Conjecture attempts to study a possible order on the set of Nash equilibria (for the specific selfish routing game we consider) that is defined with respect to their social costs; in the terminology of partially ordered sets, the FMNE Conjecture asserts that the fully mixed Nash equilibrium is a maximal element of the defined order. We feel that this order deserves further study. For example, what are the minimal elements of the order? More generally, is there a characterization of measures on Nash equilibria such that the fully mixed Nash equilibrium is a maximal element of the order defined with respect to any specific measure? (Our study considers the social cost as one such measure of interest.)

\section{Acknowledgments}

We thank Rainer Feldmann and Martin Gairing for several helpful discussions.

\section{References}

1. A. Czumaj and B. Vöcking, "Tight Bounds for Worst-Case Equilibria", Proceedings of the 13th Annual ACM Symposium on Discrete Algorithms, pp. 413-420, 2002.

2. R. Feldmann, M. Gairing, T. Lücking, B. Monien and M. Rode, "Nashification and the Coordination Ratio for a Selfish Routing Game", 30th International Colloquium on Automata, Languages and Programming, 2003.

3. D. Fotakis, S. Kontogiannis, E. Koutsoupias, M. Mavronicolas and P. Spirakis, "The Structure and Complexity of Nash Equilibria for a Selfish Routing Game,' Proceedings of the 29th International Colloquium on Automata, Languages and Programming, LNCS 2380, pp. 123-134, 2002.

4. M. Gairing, T. Lücking, M. Mavronicolas, B. Monien and P. Spirakis, "Extreme Nash Equilibria", submitted for publication, March 2003. Also available as Technical Report FLAGS-TR-02-5, Computer Technology Institute, Patras, Greece, November 2002.

5. T. Gonzalez, O.H. Ibarra and S. Sahni, "Bounds for LPT schedules on uniform processors", SIAM Journal on Computing, Vol. 6, No. 1, pp. 155-166, 1977.

6. R. L. Graham, "Bounds on Multiprocessing Timing Anomalies", SIAM Journal on Applied Mathematics, Vol. 17, pp. 416-426, 1969.

7. E. Horowitz and S. Sahni, "Exact and aproximate algorithms for scheduling nonidentical processors", Journal of the Association of Computing Machinery, Vol. 23, No. 2, pp. 317-327, 1976.

8. E. Koutsoupias, M. Mavronicolas and P. Spirakis, "Approximate Equilibria and Ball Fusion", Proceedings of the 9th International Colloquium on Structural Information and Communication Complexity, 2002, accepted to Theory of Computing Systems.

9. E. Koutsoupias and C. H. Papadimitriou, "Worst-case Equilibria", Proceedings of the 16th Annual Symposium on Theoretical Aspects of Computer Science, LNCS 1563, pp. 404-413, 1999. 
10. M. Mavronicolas and P. Spirakis, "The Price of Selfish Routing", Proceedings of the 33rd Annual ACM Symposium on Theory of Computing, pp. 510-519, 2001.

11. H. Moulin and L. Vial, "Strategically Zero-Sum Games: The Class of Games whose Completely Mixed Equilibria Cannot be Improved Upon", International Journal of Game Theory, Vol. 7, Nos. 3/4, pp. 201-221, 1978.

12. J. F. Nash, "Equilibrium Points in N-Person Games", Proceedings of the National Academy of Sciences, Vol. 36, pp. 48-49, 1950.

13. J. F. Nash, "Non-cooperative Games", Annals of Mathematics, Vol. 54, No. 2, pp. 286-295, 1951.

14. M. J. Osborne and A. Rubinstein, A Course in Game Theory, MIT Press, 1994.

15. C. H. Papadimitriou, "Algorithms, Games and the Internet", Proceedings of the 33rd Annual ACM Symposium on Theory of Computing, pp. 749-753, 2001.

16. T. E. S. Raghavan, "Completely Mixed Strategies in Bimatrix Games", Journal of London Mathematical Society, Vol. 2, No. 2, pp. 709-712, 1970. 